\title{
Effect of Co-phasing Transmission Diversity on CDMA Downlink Capacity
}

F. Tong, I.A. Glover, S.R. Pennock, P.R. Shepherd and R.C. Atkinson

This letter examines the impact of distributed antennas using co-phasing transmission diversity on downlink CDMA channels.

Introduction: This paper examines the impact of applying co-phasing transmission techniques to distributed antennas. A distributed antenna can be viewed as an irregular array of antennas with large scale separation (several wavelengths) interconnected via fiber optics, for example. Multipath propagation effects are well known in digital radio systems; multiple transmission paths between transmit and receive antennas result in both constructive and destructive interference at the receive antenna. Significant destructive interference may result in considerable amplitude attenuation of the received signal. Multiple paths also infer multiple delayed versions of the transmitted signal arriving at the receive antenna; each of these signals will be modified by the gain (magnitude \& phase) of its particular path. Where multiple transmit antennas are used to transmit to a single receive antenna, the output of each antenna must be time shifted to compensate for the different delays inherent in the different paths between each antenna pair.

Mobile radio channels are generally characterised as frequency selective fading channels: each of the frequency components in the composite waveform will be attenuated and phase shifted differently. Co-phasing implies pre-compensation for phase changes during 
transmission in order to maximise the likelihood of constructive interference and hence improving the overall signal to interference ratio (SIR).

Channel Characterisation: The impulse response of the channel, for a given user, is provided by the sum of the impulse responses from each transmit antenna. Assuming $\mathrm{K}$ transmit antennas the overall impulse response is given by:

$$
h_{c}(t)=\sum_{i=1}^{K} e^{-j \theta_{i}} h_{i}\left(t-\tau_{i}\right)
$$

hc(t): Channel impulse response

$h_{i}(t):$ Impulse response component attributable to antenna $i$

$\tau_{i}$ : Delay adjustment at antenna $\mathrm{i}$

$\theta_{\mathrm{i}}$ : Phase adjustment at antenna $\mathrm{i}$

In multi-user systems such as CDMA the distributed antenna is used to transmit to a number of users located at different geographical locations. Therefore the time and phase adjustment is also a function of the user. In a system with M mobile users, a KxM channel matrix, G, can be defined:

$$
G=\left[\begin{array}{ccc}
g_{11} & \ldots & g_{1 M} \\
\ldots & & \ldots \\
g_{K 1} & \ldots & g_{K M}
\end{array}\right]
$$


Where $g_{i j}$ represents the (power) gain between antenna I and user $\mathrm{j}$, and hence $\sqrt{g_{i j}}$ is the voltage gain between the pair.

Transmission power is distributed across the multiple antennas in accordance with the gain of the channel for that antenna; this ensures that a greater proportion of the power is transmitted from antennas that will experience the lowest attenuation. Note this approach is different from an equalisation scheme which would attempt to ensure equal power is received by from all antennas by allocating the greatest power to the weakest paths. Thus a corresponding power allocation matrix can be defined:

$$
Q=G^{T}=\left[\begin{array}{ccc}
g_{11} & \cdots & g_{K 1} \\
\cdots & & \cdots \\
g_{1 M} & \cdots & g_{K M}
\end{array}\right]
$$

The voltage induced at the receive antenna of user $\mathrm{j}$ by a signal from antenna $\mathrm{i}$ is given by the product of the channel gain and a weighting factor which is proportional to that gain, and is given by:

$$
v_{i j}=\sqrt{g_{i j}} \sqrt{g_{i j}}=g_{i j}
$$

The total voltage induced in the receive antenna of user $\mathrm{j}$ from all $\mathrm{k}$ transmit antennas is given by: 


$$
v_{j}=\sum_{i=1}^{k} g_{i j}
$$

Where $\mathrm{k}$ is the number of transmit antennas. It is apparent that this is the summation of column $\mathrm{j}$ of the power allocation matrix as shown in eqn. (3) and represents all the signal components delivered to a particular user. The power loss associated with these signal components is the square of the total voltage.

$$
\operatorname{Pr}_{j}=\left(\sum_{i=1}^{k} g_{i j}\right)^{2}
$$

In a multi-user system where each user is in a different location and has a different associated overall transmit power, $\mathrm{P}_{\mathrm{j}}$, then the total signal power delivered to the receive antenna of user $\mathrm{j}$ is given by:

$$
S_{j}=P_{j}\left(\sum_{i=1}^{k} g_{i j}\right)^{2}
$$

The interference power at a particular user, $\mathrm{j}$, can be derived in a similar manner. The total interference is the received power intended for every other user (i.e. all users except j) from each of the $\mathrm{k}$ transmit antennas. Examining each transmit antenna in turn, the voltage induced at the receive antenna of user $\mathrm{j}$ from a signal intended for user $\mathrm{m}$ is given by: 


$$
v_{i j}^{(m)}=\sqrt{g_{i m}} \sqrt{g_{i j}}
$$

The total voltage induced in the receive antenna of user $\mathrm{j}$ from all $\mathrm{k}$ antennas from a signal intended for user $m$ can be given by:

$$
v_{j}^{(m)}=\sum_{i=1}^{k} \sqrt{g_{i m}} \sqrt{g_{i j}}
$$

Recalling that the power loss is the square of the sum of received voltages, this translates

to:

$$
P_{j}^{(m)}=P_{m} \sum_{i=1}^{k} g_{i m} g_{i j}
$$

Where $\mathrm{P}_{\mathrm{m}}$ is the transmit power associated with user $\mathrm{m}$.

For a system with $\mathrm{M}$ users and orthogonal channels, the total interference experienced by user $\mathrm{j}$ is given by the summation of the interference power from all other M-1 users, and is given by:

$$
I_{j}=\sum_{m=1, m \neq j}^{M} P_{j}^{(m)}=\sum_{m=1, m \neq j}^{M} P_{m} \sum_{i=1}^{k} g_{i m} g_{i j}
$$

In cases where the CDMA channels are not truly orthogonal an inter-channel interference component may be present. The inter-channel interference may be as a result of CDMA 
codes that are not truly orthogonal or due to multipath effects; a loss factor, $\varsigma$ could be introduced:

$$
I_{j}=\varsigma\left(\sum_{m=1, m \neq j}^{M} P_{m} \sum_{i=1}^{k} g_{i m} g_{i j}\right)
$$

However, this analysis does not consider inter-channel interference; by assuming $\varsigma=1$ throughout comparisons of performance are still valid.

$$
I_{j}=\sum_{m=1, m \neq j}^{M} P_{m} \sum_{i=1}^{k} g_{i m} g_{i j}
$$

In cases where there is a single transmit antenna and single receive antenna the path loss (or channel gain) suffered by the signal $S_{j}$ and the interference $I_{j}$ will be equal. The signal to interference ratio on the downlink would then be determined entirely by the transmit power values associated with each channel (user). If the transmit power across users is uniform then the SIR at each user will also be uniform since all interference is multi-user interference that will suffer from the same path loss (gain) as the desired signal. For example, in a CDMA system with $M$ users the SIR is given by $\frac{1}{M-1}$ for uniform transmit power.

In cases where there are multiple transmit antennas uniform SIR across receivers cannot be attained simply by equalising the transmit power to all users. The difference in this 
case is that each antenna-user pair will have a distinct path loss (gain) and the overall SIR will be influenced by each of these gains ${ }^{1}$. However, uniform SIR is possible if the transmit power for each user is distributed across antennas appropriately as defined by the power allocation matrix Q. The appropriate values of Q can be determined via Eigen value decomposition; however to do this the expressions for signal power (eqn. 6) and for interference (eqn. 13) are first expressed in matrix form.

From eqn. 6 which represents the power loss associated with the signal of interest, a diagonal matrix of power loss values can be defined $D=\operatorname{diag}\left\{\operatorname{Pr}_{j}\right\}$ and from eqn. 7 a $1 \mathrm{X}$ $\mathrm{M}$ column vector of transmit powers can be defined $\mathrm{P}=\left[\mathrm{P}_{\mathrm{j}}\right]$. Thus eqn. 7 can be expressed as $1 \mathrm{X}$ M column vector $\mathrm{S}=\mathrm{PD}$. A matrix expression for interference in eqn. 13 can be derived by recognising that the interference received by a user, $\mathrm{j}$, is the sum of all transmission powers from all antennas not meant for that particular user.

An inter-user interference matrix can be determined by multiplying the power allocation matrix, $\mathrm{Q}$, by the channel gain matrix, $\mathrm{G}$, to give the weight channel gain matrix, L; where $\mathrm{L}$ is an M X M matrix such that Lij represents the aggregated loss (gain) experienced by inter-user interference experienced by user i from a signal intended for user $\mathrm{j}$ as shown in eqn. 14.

\footnotetext{
${ }^{1}$ The channels gains associated with a particular user are the elements of a particular column of eqn. 2.
} 


$$
L=\left[\begin{array}{cccc}
L_{11} & L_{21} & \ldots & I_{M 1} \\
L_{12} & L_{22} & \ldots & L_{M 2} \\
\ldots & \ldots & \ldots & \ldots \\
L_{1 M} & L_{2 M} & \ldots & L_{M M}
\end{array}\right]
$$

Then by removing the meaningless components in the leading diagonal since a user cannot interfere with itself; this gives the inter-user interference matrix I. Where $I=L-\Lambda$, and $\Lambda=\operatorname{diag}\left\{\mathrm{L}_{\mathrm{mm}}\right\} ;$ hence:

$$
I=P\left[\begin{array}{cccc}
0 & L_{21} & \ldots & I_{M 1} \\
L_{12} & 0 & \ldots & L_{M 2} \\
\ldots & \ldots & \ldots & \ldots \\
L_{1 M} & L_{2 M} & \ldots & 0
\end{array}\right]
$$

It is apparent that the process of removing the elements of the main diagonal is comparable with not considering the case when $m \neq j$ in eqn. 11 .

An expression for signal to interference ration can finally be derived as follows:

$$
\Gamma=\frac{S}{I}=\frac{P D}{P(Q G-\Lambda)}=\frac{D}{(Q G-\Lambda)}
$$

Where

$$
\Gamma=\left[\gamma_{j}\right]=\frac{S_{j}}{I_{j}}=\frac{P_{j}\left(\sum_{i=1}^{k} g_{i j}\right)^{2}}{\sum_{m=1, m \neq j}^{M} P_{m} \sum_{i=1}^{k} g_{i m} g_{i j}}
$$


Eigenvalue decomposition can be facilitated by taking the reciprocal of eqn. 16 and letting $Z=\frac{1}{\Gamma}$; this can be thought of as the interference to signal ratio:

$$
Z=(Q G-\Lambda) D^{-1}
$$

The appropriate transmission powers which give rise to uniform SIR values across users can be determined by solving eqn. 19 .

$$
P Z=\lambda P
$$

The best solution is given be the smallest eigenvalue, $\lambda_{\min }$, (and its associated eigenvector); the SIR that will be experienced by all users is given by $\gamma=\frac{1}{\lambda_{\min }}$. 